\title{
Integrating lifestyle approaches into osteoarthritis care
}

This article was published in the following Dove Press journal:

Journal of Multidisciplinary Healthcare

15 September 2015

Number of times this article has been viewed

\author{
Matthew J Garver' \\ Brian C Focht ${ }^{2}$ \\ Sarah J Taylor ${ }^{3}$ \\ 'Department of Kinesiology \\ and Nutrition, Abilene Christian \\ University, Abilene, TX, 'Department \\ of Human Sciences, Ohio State \\ University, Columbus, $\mathrm{OH},{ }^{3} \mathrm{School}$ of \\ Occupational Therapy, Texas Woman's \\ University, Dallas, TX, USA
}

Correspondence: Matthew J Garver Department of Kinesiology and Nutrition, Student Recreation and Wellness Center Room 259, Abilene Christian University, Box 28084, Abilene, TX 79699, USA

Tel +I 3256742822

Email mjgl la@acu.edu
Abstract: As the lifetime risk, societal cost, and overall functional impact of osteoarthritis (OA) is imposing, it is imperative that clinicians provide an individualized care model for patients. Patients must be offered a multiplicity of care strategies and encouraged to embrace lifestyle approaches for self-managing the effects and symptoms of OA. Certainly, the attitude of the clinician and patient will directly influence receptivity and implementation of lifestyle approaches. This work proposes how the use of structured and routine assessments and cognitive therapy ideologies may complement a comprehensive treatment plan. Assessments described herein include objective and/or self-report measures of physical function, pain, attitude about social support, and sleep quality. Baseline assessments followed by systematic monitoring of the results may give patients and clinicians valuable insight into the effectiveness of the care plan. Empirical evidence from randomized trials with OA patients highlights the effectiveness of cognitive behavioral change strategies for addressing salient concerns for OA (pain control, mobility performance, and sleep quality). Cognitive restructuring can provide patients with renewed power in managing their disease. Cognitive therapy topics discussed presently include: 1) what is OA?, 2) effectiveness of exercise and FITT (frequency, intensity, time, and type) principles for OA patients, 3) goal-setting and barriers, and 4) translating to independent care. Woven within the discussion about cognitive therapy are ideas about how the results from baseline assessments and group-mediated dynamics might assist more favorable outcomes. There are a plethora of assessments and cognitive therapy topics that could be utilized in the care strategy that we are promoting, but the present topics were selected for their low clinician and patient burden and promising results in trials with OA patients. Clinicians who are comfortable and knowledgeable about a wider range of management tools may serve more effectively in the critical, central management process and help patients embrace personal care more successfully.

Keywords: osteoarthritis, lifestyle approaches, assessments, cognitive behavioral therapy

\section{Introduction}

Osteoarthritis $(\mathrm{OA})$ can be defined through radiographic or symptomatic criteria, with joint-space narrowing and localized pain being respective hallmarks. In addition to changes in bone topography and the surrounding joint structures, pain is a primary culpable factor for associated functional decline. Deleterious risk factors consistently associated with diagnosis of incident knee OA include: body mass index (BMI), previous knee injuries, female sex, and Heberden's nodes/hand OA. ${ }^{1}$ Additionally, age, genetics, chronic occupational loading, malalignment, bone mineral density, and hormonal changes are implicated as well. ${ }^{2}$ Even among established risk factors (eg, BMI), there might be underlying considerations that mitigate or exacerbate the relationship between the given factor and the risk for OA. For instance, how strongly 
$\mathrm{BMI}$ is related to the incidence, and/or progression of OA might correlate with the age at which weight gain occurs and/ or the peak body weight during life. ${ }^{3}$

The statistics associated with lifetime risk and overall functional impact of $\mathrm{OA}$ are imposing. The lifetime risk for OA approaches $40 \%$ and $50 \%$ in men and women, respectively. ${ }^{4}$ Globally, the number of persons affected solely by knee OA has been approximated at 250 million, ${ }^{5}$ and nearly two-thirds of those affected by OA are of working age. ${ }^{6}$ Recently, the relationship between weight status and magnitude of mobility impairment has been highlighted. ${ }^{7-9}$ Moreover, mobility impairment (defined as issues with walking and climbing stairs) attributable to knee OA is greater than any other medical condition among older adults. ${ }^{10}$

\section{Pharmaceutical investment in osteoarthritis}

Despite focused investment in pharmaceutical treatments for OA, several Phase II and III clinical trials have recently failed to produce meaningful outcomes. ${ }^{1-13}$ Research and development is mired with complications, such as lack of end-point criteria and qualified biomarkers. The cost of drug development (although debatable) ${ }^{14}$ is staggering: reported to be in excess of US\$800 million. ${ }^{15}$ The combined cost and lack of success in producing a drug that concurrently promotes disease modification and significant pain relief is discouraging. Frontline pain is often controlled through paracetamol or nonsteroidal anti-inflammatory drugs (NSAIDs). However, complications of long-term NSAID usage are well documented, ${ }^{16}$ and a 2015 publication from Roberts et al raises important questions about the safety of paracetamol usage as well. ${ }^{17}$

\section{Potential framework for managing osteoarthritis}

It is obvious that individualized care is vital to patients who must be prompted toward embracing a multiplicity of self-care strategies. In light of the burgeoning increase in obesity, rapid expansion of the aged demographic, and lack of pharmaceutical options, the need for lifestyle approaches - interventions that bolster personal, patient locus of control - is vital. The aim of this publication is to propose one potential framework for clinical or personal use in which baseline assessments and candidate topics for cognitive therapy might be leveraged for assisting in the management of OA. The ideas presented should be viewed as complementary to a comprehensive treatment option.
The core of ideas described herein relates directly to successful implementation of the strategies within recent, randomized controlled trials with OA patients. ${ }^{18-20}$ The intent is to present one informed conceptualization of how OA management might be addressed through the translation of research evidence to clinical practice. This work should be regarded as a lifestyle-management approach framework in which the baseline assessments and cognitive therapy topics discussed are exchangeable with ideas from other empirical evidence. The authors are quick to point out that a systematic review of the literature would certainly reveal additional baseline assessments and cognitive therapy topics that could be used in tandem with or in place of the topics discussed. Essentially, these ideas are offered as a tool for the wide-ranging skill set that clinicians might utilize or patients might embrace.

\section{Brief on stepped care}

Wadden and Osei were early proponents of a stepped-care approach to treating patients with $\mathrm{OA},{ }^{21}$ and their tailored plan included such considerations as knowledge of current chronic disease risk factors and assessment of motivation toward dietary and weight-loss options. Adaptations of the stepped-care approach have been proposed as a resource for clinicians to utilize when treating OA patients with obesity. $^{22}$

More recently, Smink et al developed a stepwise procedure to maximize the sequencing and timing of nonsurgical strategies (BART [Beating osteoARThritis]) for OA patients. ${ }^{23}$ In this recent approach, treatment was tiered into self-care approaches (as a first step), exercise therapy, dietary considerations, and use of NSAIDs (second-step options), and more aggressive third-step approaches in the face of persisting pain (injections, electrical stimulation, and multidisciplinary care).

\section{Packaged care for osteoarthritis patients}

The process of stepped care highlights the use of more focused and intensive treatment modalities after attempts with lower-tier modalities have effectively failed to produce intended changes. Speculatively, simultaneous use of modalities apportioned to the same or different levels of stepped care may result in better long-term care. European League Against Rheumatism (EULAR) recommendations for the core management of hip and knee OA include five interventions delivered as an integrated package rather than singularly or 
in sequence. ${ }^{24}$ Recommendations include providing patients with information and education about the disease, knowledge about how to address maintenance and pacing of activities, knowledge about the development and maintenance of an individualized exercise program, education regarding weight loss (if necessary), and options to adjust mechanical loading (eg, footwear or assistive devices).

Although not necessary for diagnosis of symptomatic $\mathrm{OA}$, many practitioners report the desire to have radiographic evidence to aid in patient discussion and to ascertain the potential need for referral to a specialist. The desire to support patient discussion with visible evidence highlights a vital factor: attitudes of the patient and the practitioner toward treatment will certainly influence the choice of treatment and what lifestyle-management approaches might be employed. Packaged care may give patients greater autonomy in management of their disease.

\section{Guiding lifestyle approaches: potential usefulness of baseline assessments}

The literature has shown that clinicians who are more isolated, those who practice alone, or those who have negative views toward recommended modalities are likely to 1) embrace and offer fewer services to patients, 2) show more aggressive treatment plans, and 3) show lower levels of competence in practice. ${ }^{25,26}$ Baseline and follow-up assessment results may provide telling information for the patient and/or clinician about the effective or ineffective use of certain care strategies. In this manner, baseline assessments could become important time-point markers on which to gauge change in clinical disease state, the efficacy of treatment options, and the magnitude of change in outcomes. In addition, it may delineate the fact that there are responders and nonresponders to the care and sequence chosen; there certainly is not a single prescription to best manage the disease.

One concept that is missing in OA literature is a standardized set of valid, reliable, and agreed-upon baseline assessments. As noted earlier, this work does not systematically review the plethora of topics that might serve as baseline assessments. Rather, the ideas chosen for inclusion represent some of the more simplistic assessments possible with regard to patient and clinician time and facility demand. Accordingly, there may be need for a systematic review of the literature that highlights topics with potential usefulness within the framework proposed. As mentioned earlier, the following suggestions are hinged on strategies that have resulted in favorable outcomes in work with OA patients. ${ }^{18-20}$

We start with an offering of assessments that might be used in baseline and follow-up clinical visits. This is one conceptualization of potential care, and the ideas will need to be adjusted for clinic characteristics and patient needs. The results of these or similar assessments are pivotal for designing attributes related to goal-setting, which is an important component of cognitive therapy and the topic of a subsequent section. In particular, baseline results are ideal for constructing tailored and realistic discussion into personalized care or group-supported cognitive behavioral therapy intervention.

\section{Potential baseline assessments for osteoarthritis patients}

Presently, we are advocating that it would be judicious to consider measuring physical function, pain, attitude about social support, and sleep quality. Physical function and pain are two of the four core outcomes that are recommended for investigation during Phase III clinical trials - translational trials. ${ }^{27}$ We are also advocating the collection of information about social support and sleep quality, as these topics have widespread application for OA patients and the information gleaned from the data could certainly be utilized in cognitive therapy sessions. All of the currently included assessments can be implemented into clinical practice or personal care with minimal investment.

\section{Self-reported mobility performance}

For patients with hip and knee OA in particular, documentation of mobility performance is paramount. This type of data would evidence "activities" defined in the World Health Organization's International Classification of Functioning, Disability and Health model as germane for patients with lower-body OA. ${ }^{28}$ In addition, the discussion of results related to objective or subjective mobility performance would be important for framing cognitive therapy and shaping realistic outcome expectancies, which might occur in a group context and constitute a multidisciplinary approach to OA care.

One option for assessing self-reported physical function is the Walking Impairment Questionnaire, which has been evaluated for psychometric properties in knee OA patients. ${ }^{29}$ The measure includes 14 questions about difficulty with walking distance, walking speed, and stair-climbing ability. Participants answer on a Likert scale ranging from 4 (none) to 0 (unable to do), and the questions can be completed in 
a matter of minutes. Self-reported Walking Impairment Questionnaire outcomes for walking distance and walking speed were found to correlate with 6-minute walk distance $(r=0.52, r=0.51$, respectively) and self-reported stairclimbing ability correlated with an objective measure of stair-climbing ( $r=0.44)$.

A newer, innovative option includes the Mobility Assessment Tool-short form (MAT-sf) developed by Rejeski et al. ${ }^{30}$ Psychometrically sound, the measure uses video animations to create a standardized representation of task performance, and it improves upon the prediction of performance-based measures of function. The MAT-sf takes only a few minutes to complete. The clinical utility of the MAT-sf has been established, ${ }^{31,32}$ and it has recently been reported that baseline MAT-sf scores effectively identify risk for major mobility disability in older adults. ${ }^{33}$ A simple assessment with the MAT-sf could serve clinician and patient well and lead to structured intervention to avoid loss of mobility function.

\section{Objective measures of mobility performance}

Common objective measures of mobility performance include timed walks, distance-based walks, stair-climbing tasks, up-and-go tests, and lift-and-carry tests. Readers are referred to the quality work by Bennell et al for information about the assessments. ${ }^{34}$ Herein, a $20 \mathrm{~m}$ walk test and stair-climbing task are described. These are two of the more likely options for objective assessment of lower-extremity function that could occur in most clinical, rehabilitative, or home-based settings. Moreover, these tasks are vital for independent living.

The $20 \mathrm{~m}$ walk test can be used to monitor gait speed and changes in physical function over time. Test-retest reliability, sensitivity, and procedures for the test have been described. ${ }^{35}$ In effect, it is important that the participant has appropriate footwear on and that a clear stretch of hallway is chosen. The participant should be given one practice attempt at the walk to mitigate the learning effect. Thereafter, the participant should be timed while walking the $20 \mathrm{~m}$ course. A standard script, which has been provided by Motyl et al, ${ }^{35}$ can be used to standardize the description and testing procedures. The initial data would be personally important for comparison of future testing results.

Procedures for the stair-climbing task vary in the literature. The test will be modified in clinical or personal settings according to the physical location available, and step height and location of railing will alter outcome time (for a set climbing task) or total step number (for a timed test). Generally, the test measures a vital functional skill and assesses lower-body strength along with power and balance. As climbing tasks are common outcome goals after surgery and rehabilitation, objective documentation may support discussion of the efficacy of treatment choices, approaches toward cognitive therapy, or dialog in the group-intervention context. One method of conducting the stair-climbing task is to time the performance task (in seconds) as it relates to ascending and descending a set of stairs while walking around a centrally located handrail. ${ }^{7}$ Participants should be instructed to walk the entire time but complete the task as quickly as possible while ensuring safety. Lower completion times represent more favorable mobility performance. Stair-climbing, albeit under different procedural steps, has been found to have excellent test-retest reliability and correlate with knee strength and self-reported ambulatory disability. ${ }^{36}$

\section{Self-reported perceptions of pain}

Addressing pain management is undeniably a hallmark of OA care. A reliable assessment of pain is foundational for documenting current pain status and also for assessing the trajectory of pain and its severity over time. ${ }^{37}$ Its interference with physical function and strong influence on emotional health positions it as a leading topic for cognitive behavioral therapy. The gold standard for pain assessment remains self-report, but objective measures of pain have value, particularly when cognitive impairments may hinder memory, detection of pain, or communication about pain symptoms and severity. ${ }^{38}$ An idea conveyed in the work of Herr and Garand has application to many clinicians: "At this time, the key for practice is to find an assessment tool that patients can easily use and consistently use with each assessment". 39

The Brief Pain Inventory (BPI) was originally developed to measure pain in cancer patients. ${ }^{40} \mathrm{~A}$ valid and reliable short form has been developed as a generic measure of pain, which can apply to a wide range of OA patients. ${ }^{41}$ The responsiveness of the assessment over time and after surgery is documented. ${ }^{42}$ The duality of the measure to assess both sensory (intensity of pain) and reactive (interference of pain in the patient's life) dimensions helps to encapsulate two important components of pain. The Brief Pain Inventoryshort form (BPI-sf) allows patients to select pain ratings on a scale ranging from 0 to 10 and the word-descriptor anchors vary by question. The tool is time-efficient and endorsed by an interdisciplinary expert group. ${ }^{43}$ The BPI-sf is a solid 
choice for gathering a patient's self-assessment of pain and its interference with functional activity.

\section{Ideas about objective assessments of pain}

When cognitive impairment is suspected or documented, an objective measure of pain may be necessary and may serve in tandem with or in place of a self-report assessment. ${ }^{43}$ According to an original investigation, the Abbey Pain Scale and Pain Assessment in Advanced Dementia tool appeared to have better psychometric properties when assessing pain in a group of elderly patients enrolled in an exercise program when compared with the Pain Assessment Checklist for Seniors with Limited Ability to Communicate and the Discomfort Scale-Dementia of Alzheimer Type measures. ${ }^{44}$ Nevertheless, the complexity of considerations precludes the recommendation of one specific measurement tool for collecting objective measures of pain for persons experiencing a range of impairments in cognitive ability.

\section{Patient attitudes about social support and sleep quality}

The importance of social support and social ties to mental and physical health cannot be overemphasized. ${ }^{45,46}$ One intentional directive of group-mediated intervention is the inclusiveness and collaborative engagement that group dynamics can promote. Gauging how patients feel about their proximal support system can guide individualized, personal care (and how that might unfold within cognitive therapy and/or groupmediated intervention). One assessment tool that might provide a solid basis for understanding a patient's perception about their social support is the Social Provisions Scale. ${ }^{47}$ This contains six subscales (social integration, support for attachment, reassurance of worth, opportunity for nurturance, reliable alliance, and guidance) and 24 total items. There are four items in each subscale: two are positively formulated (presence of that type of support) and two are negatively formulated (absence of that type of support). Summing the subscales (some being reverse-coded) and summing all items provides insight into interpretation of the results. ${ }^{47}$

While it may seem misplaced, an assessment about sleep quality may provide invaluable insight into the transitory or continuous reporting of exacerbated pain symptoms and/ or compromises in function reported by patients with $\mathrm{OA}$. Moreover, it is certainly a valuable topic for discussion within multidisciplinary care. One tool for use in clinical practice is the Pittsburgh Sleep Quality Index. ${ }^{48}$ The measure has seven components (19 total items) that assess subjective sleep quality, sleep latency, sleep duration, habitual sleep efficiency, sleep disturbances, use of sleeping medications, and daytime dysfunction. Five of the items, which are to be completed by a bed partner, are not used in scoring. Overall, scores can range from 0 to 21, and higher scores indicate better sleep quality. The self-administered measure has both Likert and free-entry responses and takes approximately 5-10 minutes to complete.

\section{Guiding lifestyle approaches: potential usefulness of cognitive behavioral therapy}

Baseline assessments serve as key determinants of the effectiveness of intervention strategies. Along these lines, there is strong and amassing evidence that cognitive behavioral change strategies can invigorate and enliven patients and facilitate lifestyle changes more effectively than basic treatment or control scenarios among OA patients. For example, Focht et al have recently published works describing how to effectively deliver cognitive behavioral therapy in a group context to knee OA patients, ${ }^{18}$ and the results of the groupmediated cognitive behavioral approach were superior to a traditional exercise approach when investigating engagement in physical activity, performance of mobility functions, and assessment of physical health via the RAND-36 Physical Health Summary Scale. ${ }^{19,49,50}$

Moreover, there is evidence that cognitive behavioral therapy may influence sleep quality in OA patients. ${ }^{20,51}$ Patients undergoing cognitive behavioral therapy had greater reductions in waking after sleep onset when compared with patients receiving a placebo behavioral desensitization regimen. ${ }^{20}$ While both groups reported less pain over the course of the trial, reductions in waking after sleep onset predicted decreases in pain, and the effect was greater for those enrolled in the cognitive behavioral therapy group. ${ }^{20}$ Widespread pain is associated with disturbed, sleep and literature suggests that the relationship between the two is bidirectional. ${ }^{52}$ Accordingly, cognitive therapy may serve to provide patients with a management technique for a core distressing outcome of OA.

\section{Potential cognitive behavioral therapy and group-mediated delivery ideas for use with osteoarthritis patients}

The following sections identify four cognitive therapy topics that have widespread application across the span of patients affected by OA. Again, a systematic review of the 
literature might highlight additional topics that could be used in tandem with or in place of the topics discussed. It has been crafted based on EULAR and expert-consensus recommendations, ${ }^{24,53}$ and the successful implementation of the topics into research with OA patients. ${ }^{18-20}$ The results from baseline assessments (such as those mentioned earlier) can be purposefully leveraged in these discussions about lifestyle approaches to managing OA. EULAR has recognized the value of packaged care for patients, and they have noted core components in management. ${ }^{24}$ In addition, the benefits of group-mediated delivery can foster change through group cohesiveness, modeling, mastery experience, and increases in self-efficacy. Fundamental topics that should be addressed with each patient include: what is OA?, effectiveness of exercise and FITT (frequency, intensity, time, and type) principles for OA, goal-setting and barriers, and translating to independent care.

\section{What is osteoarthritis?}

Providing information and education about $\mathrm{OA}$ is an important and recommended first step in helping patients see different lifestyle approaches for self-managing their disease. ${ }^{24}$ Providing factual knowledge might help patients refocus their mind-set toward areas of disease management over which they have control (providing an internal locus of control). It is also important to discuss barriers (both real and perceived) and realistic outcomes for common questions related to exercise, diet, and weight loss. The options for dissemination might include but are not limited to direct conversation, educational pamphlets, telephone conversations, or web resources. There is a volume of information that might be covered in this area, but certainly there are central ideas about risk factors for the disease, how body weight impacts functional ability, effective physical activity choices, and appropriate choices related to medication usage.

With regard to risk factors, patients need to recognize that $\mathrm{OA}$ is not a "wear-and-tear" disease that is worsened by subsequent joint use. ${ }^{1,2}$ This line of thought might easily push a patient to embrace a sedentary lifestyle, which is the opposite of consensus care strategies. ${ }^{24,54}$ On the contrary, safe joint usage should occur and is pivotal for supplying the aneural and avascular cartilage with healthy, cartilaginous squishing motions. In actuality, there is no evidence that pain symptoms increase with the implementation and practice of a structured exercise program. ${ }^{55}$ Weight status has been demonstrated to be a crucial factor related to the functional ability of OA patients. Garver et $\mathrm{al}^{7}$ and Schoffman et $\mathrm{al}^{56}$ have provided information in this area. Some patients may

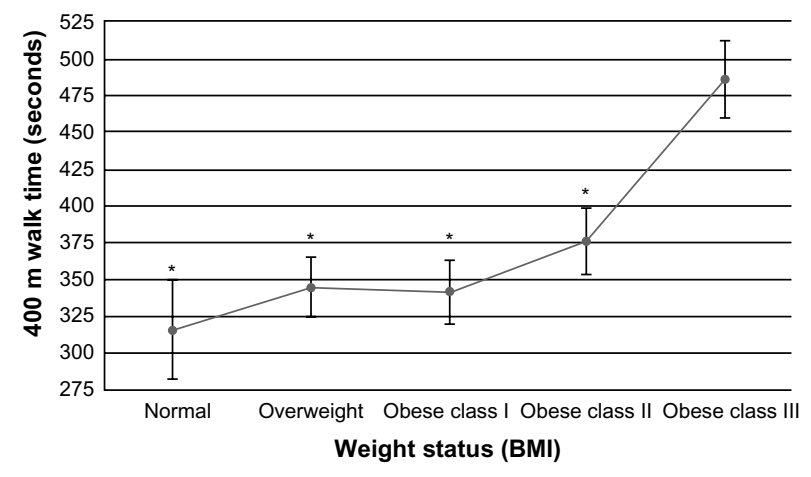

Figure I Mean ( \pm standard error) $400 \mathrm{~m}$ walk times for participants of different weight status. Weight status (BMI) of normal, overweight, obese class I, obese class II, and obese class III.

Notes: *Statistical difference from obese class III. Reproduced from Garver MJ, Focht BC, Dials J, et al.Weight status and differences in mobility performance, pain symptoms, and physical activity in older, knee osteoarthritis patients. Arthritis. Epub 2014 May $25 .^{7}$ Abbreviation: BMI, body mass index.

benefit by seeing graphs or figures about how weight status may influence mobility performance, and an example is shown in Figure 1.

The discussion of exercise should be included in the basic educational information for all OA patients, as exercise is effective for pain relief and improving function. One practical use of baseline assessments is highlighting changes in pain, function, or sleep patterns that accompany lifestyle changes. Documenting outcomes to patients at routine intervals may help a patient more fully embrace a change in their physical activity choices. Cognizance that exercise is indeed safe and furthermore that it is effective as evidenced by documented, positive, personal change is empowering. The use of paracetamol is common for managing mild-to-moderate pain, ${ }^{23,53}$ but there is evidence that patients may move quickly toward usage of NSAIDs. ${ }^{57}$ Certainly, NSAIDs may be utilized for pain that is more limiting, but they are recommended at the lowest effective dosage and not in the long term. ${ }^{53}$ Even when medication is effective at bringing pain relief, it may cause patients to feel a dependency that effectively steals their locus of control. Such a barrier may be difficult to surmount. These topics are certainly important for initial and continued management of the disease through lifestyle approaches.

\section{Effectiveness of exercise and FITT principles for OA patients}

Reviews of the literature have demonstrated the beneficial effects of exercise for reducing OA-related pain ${ }^{54,58,59}$ and helping to improve function, ${ }^{54,60}$ although the effect sizes for change in function are often not as great as those found for pain. ${ }^{54,61}$ There is some evidence that aerobic walking and leg strengthening may have similar effectiveness for pain relief 
and improvement in function, ${ }^{60}$ but other work identifies aerobic exercise as being superior. ${ }^{54}$ This may relate to that fact that the training characteristics of the ideal strength-training program have not been delineated. ${ }^{62}$ Land-based exercise appears superior to aquatic exercise for changes in pain and function. ${ }^{54}$ Additionally, class-based exercise appears more effective than home-based exercise for relieving pain. ${ }^{63}$ Individual, group-based, and home-based programs may all serve as suitable options for undertaking a program, ${ }^{24}$ but adherence (to the program chosen) is the major predictor of the long-term response to exercise. ${ }^{60}$ Working with the patients to find a program that is enjoyable, accessible, and in line with their personal goals is ideal.

Exercise is a cornerstone for OA patients, and discussion about the appropriate type of program and how to monitor characteristics of a program are important. The problem is that patients are reluctant or resistant to engage in exercise programs, and clinicians are not equipped or do not choose to provide patients with information that addresses these and other salient barriers. ${ }^{64}$ With this knowledge in mind, clinicians should encourage patients to undertake healthy lifestyle options through three deliberate behaviors.

First, patients should be taught about the connection between sedentary behavior and physical function and encouraged to reduce sedentary time. In a study investigating sedentary behavior, individuals apportioned to the most sedentary quartile were found to have the worst outcomes for a $20 \mathrm{~m}$ walk and chair stand tests, even after adjusting for demographics, health factors, and time spent in moderate or greater physical activity minutes ${ }^{65}$ The fact that sedentary behavior is independently tied to physical function is an important note to share with OA patients. ${ }^{65}$ This conversation is intended to help patients acknowledge the additive effect of simple lifestyle choices, such as spending less time in near-resting activities.

Second, patients should be taught to embrace healthy choices through active living. It is a misconception that exercise must be intense or of longer duration to be meaningful. Rather, patients need to hear that 10-minute bouts of activity several times per day can result in improvements in health. ${ }^{66}$ Informing individuals that they can "gather health" in 10-minute bouts may be a great way to restructure conversations about lifestyle approaches for managing OA. Active living can occur through selection of parking spots, taking stairs, or walking for a few minutes down the street before picking up the mail.

Third, patients need to hear about the benefits of a regular exercise program and what outcomes are realistic, and they need to be supported to adopt a structured exercise program that they enjoy. This will require that many patients be taught how to monitor the intensity of their work. Group support is particularly impactful in supporting the adoption and maintenance of a program. A basic method for framing exercise is with the FITT principle. Many patients need to be informed directly about the frequency (how many days per week they need to exercise), intensity (how much effort or discomfort they should feel), time (how long to exercise), and type (what activity to choose). It is most difficult to describe the intensity of exercise, and while heart rate is commonly used, rating of perceived exertion (RPE) ${ }^{67}$ scales exist and have been successfully used with older, overweight OA patients by Focht et al. ${ }^{18,19}$ Additionally, patients should recognize that exercise is associated with transient increases in pain ${ }^{68}$ but better long-term pain control. ${ }^{59}$ Discussions about how the right choice of exercise intensity will slightly increase pain but not cause it to linger can occur once patients understand the FITT principle. The FITT principle is also vital for assessing the practicality and attainment of short- and long-term exercise goals. With any exercise program, booster sessions (in simple, follow-up conversations) have been found to be important for long-term maintenance. ${ }^{61,69}$

\section{Goal-setting and barriers}

Patients should be informed about the importance of setting goals to address their most pertinent needs, often physical activity choices and nutritional choices with weight loss being a necessary outcome. Goals should be set to assess short-term outcomes and longer-term direction. The acronym SMART (specific, measurable, achievable, realistic, and timed) is often used to set objectives. In effect, goals must be written in a manner that allows for evidence of their attainment to be collected.

A goal toward physical activity might be, "I want to perform 20 minutes of walking and 20 minutes of legstrengthening exercise three times this week. I will work at an RPE between 11 and 13 on the Borg scale for the purposes of cardiovascular health and strengthening the musculature around the hip and knee". Assessment of the goal could be documented by self-report and corroborated by a workout partner. A goal toward nutritional choices might be, "I want to measure serving sizes of each food that I eat and drink that I consume for 4 days this week. I will record the calories of the food and drink items in a food diary and restrict myself to fewer than 1,800 calories each of those 4 days". This nutritional goal could be built upon, but one comparable to it may be an important initial step in helping a patient see how quickly calories can be consumed. 
Cognitive therapy based on how much work must be applied to burn calories is a great option once understanding of caloric intake is documented. Truthfully, it is very difficult for patients to engage in enough exercise to overcome poor dietary choices, so goals in this area may prove enlightening. Patients need to be informed that even modest weight loss can help lead to clinically meaningful improvements in function and disease symptoms. ${ }^{70,71}$ The results of baseline assessments can be purposefully utilized to guide goal-setting practices in the areas of activity and nutrition.

It is vital that patients see goal-setting as a continuous and dynamic event. Meeting goals will necessitate overcoming barriers. Therefore, the discussion of barriers (real and perceived) is indispensable. The strengths of group-mediated work (modeling and mastery experience, among others) may be especially compelling in this area as individuals set and struggle to give effort to meet personal SMART goals. The presentation and discussion of goals and barriers within the group context can be exceptionally meaningful as like-bodied persons share their individual stories of failure and success. A multidisciplinary professional approach to goal-setting and barriers may be fruitful. Registered dieticians, exercise physiologists, and psychologists, for example, may be necessary to address the full gamut of needs. Professionals may have clinical insight to help patients set realistic SMART goals and help them reframe how powerful and insurmountable a barrier might appear. Using trained professionals in a group context may also be the most effective use of time and financial resources.

\section{Translating to independent care}

The task of helping patients manage OA is fraught with challenges, not the least of which is to encourage adherence to healthy activity and nutritional choices, two undeniable cornerstones of health. Translating the onus of health care to the patient will likely require a social support structure. Social support might be thought of as a network of connections that support a specific outcome. The successful transfer of responsibility may more successfully occur in a group-mediated context, ${ }^{19}$ but skills necessary for individual success, be it in group-mediated or individual format, are largely the same. Patients will need to set goals, overcome personal barriers, and find personal support systems or community resources with similar aims (to incorporate healthy lifestyle choices that effectively manage the OA disease and its symptoms). Patients need to be encouraged to be an active part of finding, forming associations, and engaging with others who are like-bodied. If group-mediated structures can be established by patients or clinicians, or if community resources can bring together individuals looking to manage disease, these are natural and potentially strong sources of social support. As mentioned earlier, goal-setting is a dynamic process. One important goal for patients might be established regarding seeking out and choosing to get involved with a social support network.

\section{Conclusion}

Lifestyle approaches to counter the effects and symptoms of OA are vital for patients who must learn to self-manage and clinicians who must balance caring for each individual patient. As the attitude of the clinician and patient will directly influence the reception and implementation of suggested lifestyle approaches, patients should be prompted toward embracing a multiplicity of self-care strategies. Addressing patients with packaged care that utilizes baseline assessments and cognitive therapy ideologies may be a great way for patients to see their current state, the results of their management strategies over time, and be provided with simplistic means of finding support for their disease. The ideas presented should be viewed as a complement to any comprehensive treatment plan.

Systematic monitoring of baseline-testing results may give patients and clinicians valuable insight into the effectiveness of the management plan. Through this framework, clinicians can serve in a critical, central role in the care process. There is strong evidence that group dynamics can foster the acceptance and practice of cognitive behavioral change strategies, thus leading to more effective adoption and longerterm results. This option might be explored more fully by clinicians. Within personal care or group-mediated contexts, goal-setting should be encouraged. Helping patients recognize that they have power in managing their disease can have significant implications for effective self-management.

\section{Disclosure}

The authors report no conflicts of interest in this work.

\section{References}

1. Blagojevic M, Jinks C, Jeffery A, Jordan K. Risk factors for onset of osteoarthritis of the knee in older adults: a systematic review and metaanalysis. Osteoarthritis Cartilage. 2010;18(1):24-33.

2. Felson DT, Lawrence RC, Dieppe PA, et al. Osteoarthritis: new insights. Part 1: the disease and its risk factors. Ann Intern Med. 2000;133(8):635-646.

3. Sowers MR, Karvonen-Gutierrez CA. The evolving role of obesity in knee osteoarthritis. Curr Opin Rheumatol. 2010;22(5):533-537.

4. Lawrence RC, Felson DT, Helmick CG, et al. Estimates of the prevalence of arthritis and other rheumatic conditions in the United States. Part II. Arthritis Rheum. 2008;58(1):26-35. 
5. Murray CJ, Vos T, Lozano R, et al. Disability-adjusted life years (DALYs) for 291 diseases and injuries in 21 regions, 1990-2010: a systematic analysis for the Global Burden of Disease Study 2010. Lancet. 2013;380(9859):2197-2223.

6. Losina E, Weinstein AM, Reichmann WM, et al. Lifetime risk and age at diagnosis of symptomatic knee osteoarthritis in the US. Arthritis Care Res (Hoboken). 2013;65(5):703-711.

7. Garver MJ, Focht BC, Dials J, et al. Weight status and differences in mobility performance, pain symptoms, and physical activity in older, knee osteoarthritis patients. Arthritis. 2014;2014:375909.

8. Garver MJ, Focht BC, Devor ST, Dials J, Emery C, Hackshaw K. Functional performance, mobility-related self-efficacy, and physical activity in older, overweight and obese knee osteoarthritis patients. Med Sci Sports Exerc. 2011;43(5):321-322.

9. Garver MJ. Improving Maintenance of Physical ACtivity Trial - Pilot (IMPACT-P) [doctoral thesis]. Columbus (OH): Ohio State University; 2011.

10. Guccione AA, Felson DT, Anderson JJ, et al. The effects of specific medical conditions on the functional limitations of elders in the Framingham Study. Am J Public Health. 1994;84(3):351-358.

11. Chevalier X, Goupille P, Beaulieu A, et al. Intraarticular injection of anakinra in osteoarthritis of the knee: a multicenter, randomized, double-blind, placebo-controlled study. Arthritis Rheum. 2009; 61(3):344-352.

12. Chevalier X, Jerosch J, Goupille P, et al. Single, intra-articular treatment with $6 \mathrm{~mL}$ hylan GF 20 in patients with symptomatic primary osteoarthritis of the knee: a randomised, multicentre, double-blind, placebo controlled trial. Ann Rheum Dis. 2010;69(1):113-119.

13. Malemud C. Repair of injury to articular cartilage with chondrocyte progenitor cells. Rheumatol Curr Res. 2013;3(2):122.

14. Collier R. Drug development cost estimates hard to swallow. Can Med Assoc J. 2009;180(3):279-280.

15. DiMasi JA, Hansen RW, Grabowski HG. The price of innovation: new estimates of drug development costs. J Health Econ. 2003;22(2) 151-185.

16. Singh G. Gastrointestinal complications of prescription and overthe-counter nonsteroidal anti-inflammatory drugs: a view from the ARAMIS database. Am J Ther. 2000;7(2):115-122.

17. Roberts E, Delgado Nunes V, Buckner S, et al. Paracetamol: not as safe as we thought? A systematic literature review of observational studies. Ann Rheum Dis. Epub 2015 Mar 2.

18. Focht BC, Garver MJ, Devor ST, et al. Improving maintenance of physical activity in older, knee osteoarthritis patients trial-pilot (IMPACT-P): design and methods. Contemp Clin Trials. 2012;33(5): 976-982.

19. Focht BC, Garver MJ, Devor ST, et al. Group-mediated physical activity promotion and mobility in sedentary patients with knee osteoarthritis: results from the IMPACT-pilot trial. $J$ Rheumatol. 2014;41(10): 2068-2077.

20. Smith MT, Finan PH, Buenaver LF, et al. Cognitive-behavioral therapy for insomnia in knee osteoarthritis: a randomized, double-blind, active placebo-controlled clinical trial. Arthritis Rheumatol. 2015;67(5): 1221-1233.

21. Wadden TA, Osei S. The treatment of obesity: an overview. In: Wadden TA, Stunkard AJ, editors. Handbook of Obesity Treatment New York: Guilford Press; 2002:229-248.

22. Focht BC, Rejeski WJ. Managing obesity in patients who have knee osteoarthritis. 2009. Available from: http://www.rheumatologynetwork. com/osteoarthritis/managing-obesity-patients-who-have-kneeosteoarthritis. Accessed August 11, 2015.

23. SminkAJ, van den Ende CH, Vlieland TP, et al. "Beating osteoARThritis": development of a stepped care strategy to optimize utilization and timing of non-surgical treatment modalities for patients with hip or knee osteoarthritis. Clin Rheumatol. 2011;30(12):1623-1629.

24. Fernandes L, Hagen KB, Bijlsma JW, et al. EULAR recommendations for the non-pharmacological core management of hip and knee osteoarthritis. Ann Rheum Dis. 2013;72(7):1125-1135.
25. Landon BE, Reschovsky J, Reed M, Blumenthal D. Personal, organizational, and market level influences on physicians' practice patterns: results of a national survey of primary care physicians. Med Care. 2001; 39(8):889-905.

26. Lockyer JM. Physician performance: the roles of knowledge, skill, and environment. Teach Learn Med. 1992;4(2):86-96.

27. Bellamy N, Kirwan J, Boers M, et al. Recommendations for a core set of outcome measures for future phase III clinical trials in knee, hip, and hand osteoarthritis. Consensus development at OMERACT III. J Rheumatol. 1997;24(4):799-802.

28. World Health Organization. International Classification of Functioning, Disability and Health. Geneva: WHO; 2001.

29. Collins E, O’Connell S, Jelinek C, Miskevics S, Budiman-Mak E. Evaluation of psychometric properties of walking impairment questionnaire in overweight patients with osteoarthritis of knee. J Rehabil Res Dev. 2008;45(4):559-566.

30. Rejeski WJ, Ip EH, Marsh AP, Barnard RT. Development and validation of a video-animated tool for assessing mobility. $J$ Gerontol A Biol Sci Med Sci. 2010;65(6):664-671.

31. Ip EH, Rejeski WJ, Marsh AP, Barnard RT, Chen SH. Psychometric properties of a computerized adaptive test for assessing mobility in older adults using novel video-animation technology. Qual Life Res. 2013;22(8):1907-1915.

32. Rejeski WJ, Marsh AP, Anton S, et al. The MAT-sf: clinical relevance and validity. J Gerontol A Biol Sci Med Sci. 2013;68(12):1567-1574.

33. Rejeski WJ, Rushing J, Guralnik JM, et al. The MAT-sf: identifying risk for major mobility disability. J Gerontol A Biol Sci Med Sci. 2015;70(5): 641-646.

34. Bennell K, Dobson F, Hinman R. Measures of physical performance assessments: Self-Paced Walk Test (SPWT), Stair Climb Test (SCT), Six-Minute Walk Test (6MWT), Chair Stand Test (CST), Timed Up and Go (TUG), Sock Test, Lift and Carry Test (LCT), and Car Task. Arthritis Care Res (Hoboken). 2011;63 Suppl 11:S350-S370.

35. Motyl JM, Driban JB, McAdams E, Price LL, McAlindon TE. Test-retest reliability and sensitivity of the 20-meter walk test among patients with knee osteoarthritis. BMC Musculoskelet Disord. 2013;14:166.

36. Rejeski WJ, Ettinger WH, Schumaker S, James P, Burns R, Elam JT. Assessing performance-related disability in patients with knee osteoarthritis. Osteoarthritis Cartilage. 1995;3(3):157-167.

37. American Geriatrics Society Panel on the Pharmacological Management of Persistent Pain in Older Persons. Pharmacological management of persistent pain in older persons. J Am Geriatr Soc. 2009;57(8): 1331-1346.

38. Buffum MD, Hutt E, Chang VT, Craine MH, Snow AL. Cognitive impairment and pain management: review of issues and challenges. J Rehabil Res Dev. 2007;44(2):315-330.

39. Herr KA, Garand L. Assessment and measurement of pain in older adults. Clin Geriatr Med. 2001;17(3):457-478.

40. Cleeland C, Ryan K. Pain assessment: global use of the Brief Pain Inventory. Ann Acad Med Singapore. 1994;23(2):129-138.

41. Mendoza T, Mayne T, Rublee D, Cleeland C. Reliability and validity of a modified Brief Pain Inventory short form in patients with osteoarthritis. Eur J Pain. 2006;10(4):353-361.

42. Kapstad H, Rokne B, Stavem K. Psychometric properties of the Brief Pain Inventory among patients with osteoarthritis undergoing total hip replacement surgery. Health Qual Life Outcomes. 2010;8:148.

43. Hadjistavropoulos T, Herr K, Turk DC, et al. An interdisciplinary expert consensus statement on assessment of pain in older persons. Clin J Pain. 2007;23(1 Suppl):S1-S43.

44. Liu JY, Briggs M, Closs SJ. The psychometric qualities of four observational pain tools (OPTs) for the assessment of pain in elderly people with osteoarthritic pain. J Pain Symptom Manage. 2010; 40(4):582-598.

45. Uchino BN. Social support and health: a review of physiological processes potentially underlying links to disease outcomes. J Behav Med. 2006;29(4):377-387. 
46. Thoits PA. Mechanisms linking social ties and support to physical and mental health. J Health Soc Behav. 2011;52(2):145-161.

47. Russell DW, Cutrona CE. Social Provisions Scale. Ames (IA): Iowa State University; 1984.

48. Buysse DJ, Reynolds CF, Monk TH, Berman SR, Kupfer DJ. The Pittsburgh Sleep Quality Index: a new instrument for psychiatric practice and research. Psychiatry Res. 1989;28(2):193-213.

49. Focht BC, Garver MJ, Devor ST, et al. Short-term changes in pedometerdetermined physical activity in knee osteoarthritis patients: evidence from the IMPACT-P trial. Med Sci Sports Exerc. 2011;43(5):75-76.

50. Lucas AR, Focht BC, Garver MJ, et al. Physical activity and change in health-related quality of life in older, knee osteoarthritis patients. Med Sci Sports Exerc. 2012;44:85. Available from http://journals.lww.com/ acsm-msse/Fulltext/2012/05002/Abst_A_FreeCommSlides.2.aspx. Accessed September 2, 2015.

51. Vitiello MV, Rybarczyk B, Von Korff M, Stepanski EJ. Cognitive behavioral therapy for insomnia improves sleep and decreases pain in older adults with co-morbid insomnia and osteoarthritis. J Clin Sleep Med. 2009;5(4):355-362.

52. McBeth J, Wilkie R, Bedson J, Chew-Graham C, Lacey R. Sleep disturbance and chronic widespread pain. Curr Rheumatol Rep. 2015; 17(1):469.

53. Zhang W, Nuki G, Moskowitz R, et al. OARSI recommendations for the management of hip and knee osteoarthritis: part III: changes in evidence following systematic cumulative update of research published through January 2009. Osteoarthritis Cartilage. 2010;18(4):476-499.

54. Bennell KL, Hinman RS. A review of the clinical evidence for exercise in osteoarthritis of the hip and knee. J Sci Med Sport. 2011;14(1):4-9.

55. Vignon E, Valat JP, Rossignol M, et al. Osteoarthritis of the knee and hip and activity: a systematic international review and synthesis (OASIS). Joint Bone Spine. 2006;73(4):442-455.

56. Schoffman DE, Wilcox S, Baruth M. Association of body mass index with physical function and health-related quality of life in adults with arthritis. Arthritis. 2013;2013:190868.

57. Jordan K, Sawyer S, Coakley P, Smith H, Cooper C, Arden N. The use of conventional and complementary treatments for knee osteoarthritis in the community. Rheumatology (Oxford). 2004;43(3):381-384.

58. Jamtvedt G, Dahm KT, Christie A, et al. Physical therapy interventions for patients with osteoarthritis of the knee: an overview of systematic reviews. Phys Ther. 2008;88(1):123-136.

59. Focht BC. Effectiveness of exercise interventions in reducing pain symptoms among older adults with knee osteoarthritis: a review. JAging Phys Act. 2006;14(2):212-235.
60. Roddy E, Zhang W, Doherty M. Aerobic walking or strengthening exercise for osteoarthritis of the knee? A systematic review. Ann Rheum Dis. 2005;64(4):544-548.

61. Jansen MJ, Viechtbauer W, Lenssen AF, Hendriks EJ, de Bie RA. Strength training alone, exercise therapy alone, and exercise therapy with passive manual mobilisation each reduce pain and disability in people with knee osteoarthritis: a systematic review. J Physiother. 2011; 57(1):11-20.

62. Lange AK, Vanwanseele B, Fiatarone Singh MA. Strength training for treatment of osteoarthritis of the knee: a systematic review. Arthritis Rheum. 2008;59(10):1488-1494.

63. Thomas A, Eichenberger G, Kempton C, et al. Recommendations for the treatment of knee osteoarthritis, using various therapy techniques, based on categorizations of a literature review. J Geriatr Phys Ther. 2009;32(1):33-38.

64. Abramson S, Stein J, Schaufele M, Frates E, Rogan S. Personal exercise habits and counseling practices of primary care physicians: a national survey. Clin J Sport Med. 2000;10(1):40-48.

65. Lee J, Chang RW, Ehrlich-Jones L, et al. Sedentary behavior and physical function: objective evidence from the Osteoarthritis Initiative. Arthritis Care Res (Hoboken). 2015;67(3):366-373.

66. Haskell WL, Lee IM, Pate RR, et al. Physical activity and public health: updated recommendation for adults from the American College of Sports Medicine and the American Heart Association. Circulation. 2007;116(9):1081-1093.

67. Borg GA. Psychophysical bases of perceived exertion. Med Sci Sports Exerc. 1982;14(5):377-381.

68. Focht BC, Ewing V, Gauvin L, Rejeski WJ. The unique and transient impact of acute exercise on pain perception in older, overweight, or obese adults with knee osteoarthritis. Ann Behav Med. 2002;24(3): 201-210.

69. McCarthy C, Mills P, Pullen R, Roberts C, Silman A, Oldham J. Supplementing a home exercise programme with a class-based exercise programme is more effective than home exercise alone in the treatment of knee osteoarthritis. Rheumatology (Oxford). 2004;43(7):880-886.

70. Messier SP, Loeser RF, Miller GD, et al. Exercise and dietary weight loss in overweight and obese older adults with knee osteoarthritis: the Arthritis, Diet, and Activity Promotion Trial. Arthritis Rheum. 2004; 50(5):1501-1510.

71. Messier SP, Loeser RF, Mitchell MN, et al. Exercise and weight loss in obese older adults with knee osteoarthritis: a preliminary study. J Am Geriatr Soc. 2000;48(9):1062-1072.
Journal of Multidisciplinary Healthcare

\section{Publish your work in this journal}

The Journal of Multidisciplinary Healthcare is an international, peerreviewed open-access journal that aims to represent and publish research in healthcare areas delivered by practitioners of different disciplines. This includes studies and reviews conducted by multidisciplinary teams as well as research which evaluates the results or conduct of such teams or healthcare processes in general. The journal covers a wide range of areas and welcomes submissions from practitioners at all levels, from all over the world. The manuscript management system is completely online and includes a very quick and fair peer-review system. Visit http://www.dovepress.com/testimonials.php to read real quotes from published authors. 